Lengua y cultura. Reflexiones sobre la elaboración de programas para la enseñanza de las lenguas indígenas de México como L2 desde el Marco Común Europeo de Referencia para las Lenguas ${ }^{1}$

María Soledad Pérez López ${ }^{2}$

Alejandra Arellano Martínez ${ }^{3}$

\title{
RESUMEN
}

En México, de acuerdo a la legislación vigente, entre otras condiciones sociales necesarias para construir una nación efectivamente multicultural y multilingüe, es necesario garantizar la disponibilidad y el acceso de las lenguas indígenas nacionales al público general. Para esto, es fundamental que existan espacios de enseñanza y aprendizaje de este conjunto de lenguas que ayuden a formar ciudadanos plurilingües tanto en el sistema de educación nacional como fuera de él. Este artículo pretende dar cuenta de la experiencia en la elaboración de programas y materiales didácticos dirigidos a este propósito, desde la utilización del Marco Común de Referencia para las lenguas (MCRL).

PALABRAS ClAVE: Lengua. Cultura. Diseño de programas de enseñanza. Enseñanza lenguas indígenas.

\section{Linguagem e cultura. Reflexões sobre o desenvolvimento de programas para o ensino de línguas indígenas do México como L2 do Quadro Europeu Comum de Referência para Línguas}

RESUMO

No México, de acordo com a legislação vigente, entre as condições sociais necessárias para construir uma Nação nação efetivamente multicultural e multilíngue, vale destacar a disponibilidade de línguas indígenas nacionais para a população mexicana. Para isso, é fundamental que haja espaços de ensino e aprendizagem das línguas indígenas nacionais que ajudem a formar cidadãos bilíngues, tanto do sistema educacional como de fora dele. Este artigo visa dar conta de apresentar uma proposta de desenvolvimento de materiais didáticos como o Quadro Europeu Comum de Referência para as Línguas (QECR) para o fortalecimento dos programas de ensino de línguas nacionais.

PALAVRAS-CHAVE: Língua. Cultura. Desenho de programas de ensino. Ensino de línguas indígenas.

\footnotetext{
${ }^{1}$ Ponencia presentada en el Primer Congreso Internacional de Pueblos Indígenas, Oaxaca, 2013, revisada y ampliada.

2 Universidad Pedagógica Nacional (UPN), Ciudad de México, CDMX, México. E-mail: baramousso@gmail.com.

${ }^{3}$ Instituto Nacional de Lenguas Indígenas (INALI), Cidade do México, México. E-mail: alearmar@ hotmail.com
} 


\title{
Language and culture. Reflections about the syllabus design for natives language teaching from the Common European Framework of Reference for Languages
}

\begin{abstract}
In Mexico, according to current legislation, among other social conditions necessary to build an effectively multicultural and multilingual nation, it is necessary to guarantee the availability and access of national indigenous languages to the general public. For this, it is essential that there are spaces for teaching and learning of this set of languages that help to train multilingual citizens both in the national education system and outside it. This article aims to give an account of the experience in the development of teaching programs and materials aimed at this purpose, from the use of the Common Framework of Reference for languages (MCRL).
\end{abstract}

KEY WORDS: Languages. Culture. Syllabuses design. Natives languages teachings.

\section{Introducción}

En México, entre las condiciones sociales necesarias para construir una nación efectivamente pluricultural y multilingüe acorde con la legislación vigente, destaca el hacer asequibles las lenguas indígenas nacionales a la población mexicana. Para ello, es fundamental contar con espacios de enseñanza y aprendizaje de las lenguas indígenas nacionales, que ayuden a formar ciudadanos plurilingües, tanto desde el sistema educativo como fuera de éste.

El desarrollo de propuestas efectivas para la enseñanza-aprendizaje de las lenguas indígenas nacionales, se torna aún más necesario al considerar que de 15.7 millones de personas que se autoidentifican como indígenas, más de 9 millones declaran no hablar ninguna lengua indígena (INEGI, 2010). Esto representa cerca de 60\% de este sector, resultado de las políticas de castellanización que han marcado la situación sociolingüística de las lenguas nacionales.

En este campo, y en contraste con la atención que se ha dado a la enseñanza de lenguas extranjeras, el desarrollo de propuestas de enseñanza de lenguas indígenas se caracteriza por contar con una serie de experiencias aisladas, en las que los sujetos interesados en enseñar sus idiomas construyen sus propios programas a partir de su experiencia personal, ya sea con el modelo basado en su aprendizaje del español durante su trayecto escolar, o bien en su acercamiento al aprendizaje de alguna lengua extranjera -en especial inglés.

La enseñanza de lenguas indígenas en México se enfrenta también a un escenario complejo y de múltiples necesidades, como la carencia de metodologías y programas de 
enseñanza, escasos materiales didácticos y, en general, de materiales de consulta que apoyen la práctica de enseñanza-aprendizaje (diccionarios, gramáticas, etc.), así como las limitadas oportunidades de continuar en cursos "avanzados", la movilidad de los aprendices y la escasa retribución económica que reciben los instructores. A esto se suma la falta de propuestas y mecanismos de formación, actualización y evaluación de los agentes educativos que imparten dichos cursos.

Ante tal panorama, el Instituto Nacional de Lenguas Indígenas (Inali), en colaboración con la Universidad Pedagógica Nacional (UPN), ha impulsado desde finales de 2011 el proyecto "Fortalecimiento de las prácticas de enseñanza-aprendizaje de lenguas indígenas como segundas lenguas" (FPEALIL2), ${ }^{4}$ que tiene como propósito fortalecer los esfuerzos de los promotores de la lengua, quienes imparten cursos de en espacios públicos: casas de cultura, academias de lenguas indígenas y otras organizaciones civiles, para difundir y enseñar las lenguas indígenas como lengua adicional ${ }^{5}$ a publico general ${ }^{6}$ interesado en aprenderlas. Lo anterior, en la búsqueda de estrategias que permitan hacer accesible el aprendizaje de lenguas indígenas, e incrementar su prestigio social al ofrecer cursos de enseñanza cada vez más eficaces.

Para esta tarea se consideró pertinente retomar los planteamientos del Marco Común Europeo de Referencia para las Lenguas (MCERL), y valorar su "alcance" como una herramienta para la enseñanza de las lenguas indígenas nacionales. Esta consideración parte de reconocer que el MCERL es producto de una investigación sistemática sobre los resultados en enseñanza de las lenguas -elaborada por expertos en el campo de la enseñanza y el aprendizaje de idiomas- y, en ese sentido, proporciona una plataforma para reflexionar, proponer y valorar los procesos y competencias que se tienen que desarrollar para la enseñanza de cualquier idioma.

Sin embargo, su aplicación a la enseñanza de lenguas indígenas ha decantado una serie de interrogantes respecto a su funcionalidad e instrumentación que, por otro lado, sugieren un productivo debate aún pendiente en México. Estas son: ¿Puede el Marco Común Europeo de Referencia para las Lenguas (MCERL, 2002) apoyar el desarrollo de referenciales y programas

\footnotetext{
${ }^{4}$ En diferentes etapas de este proyecto se ha contado también con la valiosa colaboración de investigadores y estudiantes de la Universidad Intercultural del Estado de México y la Universidad Autónoma de Nayarit.

${ }^{5}$ Nos referimos a lenguas adicionales independientemente de que sea segunda, tercera o más pero la referimos como L2. No utilizamos el término lengua materna ya que este puede referirse a la lengua que tendría que haber sido transmitida pero no lo fue. Es en este sentido que en diversas comunidades indígenas se refieren a la lengua madre, independientemente de que se hable o no, en su lugar nos referimos a primera lengua (L1).

${ }^{6}$ Por público general nos referimos a todos aquellos sujetos que desean aprender a hablar una lengua indígena independientemente de que sea la lengua que tendrían que haber heredado o no.
} 
para las lenguas nacionales?; en su instrumentación, ¿cómo evitar una simple proyección de las características de las lenguas y los contextos sociolingüísticos en que fue desarrollado?; ¿cómo respetar las prácticas lingüísticas en que tienen funcionalidad las lenguas indígenas y, a la vez, proyectar ámbitos de uso más amplios?; ¿cómo hacer de la enseñanza de las lenguas indígenas una fuente de enriquecimiento cultural para el país?

Para revisar estas preguntas se aborda, en primer lugar, la forma en que el MCERL plantea sus orientaciones para diseñar programas de enseñanza, después, cómo se han utilizado dichas herramientas y las implicaciones que ha tenido retomar las particularidades culturales en los programas de lengua, y finalmente se presentan algunas consideraciones y retos que plantean el diseño de los programas de enseñanza de lenguas indígenas nacionales, desde nuestras experiencias.

\section{El Marco Común Europeo de Referencia para las Lenguas}

Entre los aspectos útiles que plantea el MCERL para elaborar, mejorar o complementar los programas de enseñanza para las lenguas nacionales, se destacan cuatro puntos:

1. Considera una estructura gradual y progresiva para el aprendizaje de la lengua, mediante una serie de descriptores ${ }^{7}$ que sugieren los objetivos que se espera desarrolle el estudiante en cada nivel. Con ello se conforman los "niveles comunes de referencia”, los cuales aportan orientación sobre qué se enseña y qué se evalúa.

2. Orienta la comprensión del aprendizaje de una lengua desde un enfoque accional, que busca el desarrollo de una competencia comunicativa, definida a partir de tres componentes:

- La competencia sociolingüística o pragmática: el conocimiento de las reglas de la comunicación de la cultura y de las estrategias para conseguir comunicarse.

- La competencia lingüística: el conocimiento de la lengua como sistema (léxico, gramática, fonología).

- La competencia cultural: lo que concierne al conocimiento de los países en los que las lenguas son habladas y las culturas que les son propias, sin lo cual la

\footnotetext{
${ }^{7}$ De manera general, se comprende como los criterios que definen lo que "Puede hacer" el usuario de la lengua en cuanto a actividades comunicativas, las estrategias empleadas en la realización de actividades comunicativas, y las competencia lingüística, pragmática y sociolingüística, en un esquema escalonado. Instituto Cervantes (2002). Marco común europeo de referencia para la enseñanza de lenguas. Enseñanza aprendizaje y evaluación.
} 
comunicación no podría establecerse.

3. Reconoce a los usuarios y aprendices de una lengua como agentes sociales, por lo cual ponen en ejercicio su competencia comunicativa al realizar actividades de la lengua, como son:

- La comprensión (oral y, en su caso, escrita).

- La expresión (oral y, en su caso, escrita).

- La interacción (supone un intercambio entre dos o más personas).

- La mediación (para hacer posible la comunicación entre personas incapaces de comunicarse directamente entre sí, como actividades de traducción y de interpretación).

Y asume que todas estas actividades se realizan en un contexto o ámbito público, personal, profesional o educativo.

4. Plantea que la comunicación y el aprendizaje supone la realización de tareas, comprendida como el despliegue de las estrategias y habilidades que posee una persona para lograr algo -desde lo más pragmático (construir un mueble siguiendo un instructivo), hasta lo más conceptual (escribir un libro, argumentar, llevar a cabo una negociación)-, colocando el uso de la lengua asociado intrínsecamente a las acciones ejecutadas por un sujeto (Eduscol, 2011).

Tales consideraciones permiten acercarse a una perspectiva en la enseñanza de lenguas más allá de la memorización de listas de palabras o reglas gramaticales, hacia el desarrollo de habilidades para entablar una conversación.

El MCERL, como menciona, tiene el propósito de ofrecer a los profesionistas en la materia un conjunto de "principios prácticos para la enseñanza, el aprendizaje y la evaluación de lenguas", en ese sentido no pretende establecer ni objetivos para los usuarios de una lengua, ni métodos para lograrlos. En todo caso, especifica que sea abierto y flexible a las adaptaciones necesarias. Así, el MCERL es sólo un instrumento que determina descriptores generales sobre los cuales cada lengua tendrá que establecer los rasgos particulares e identificar las realizaciones correspondientes a cada lengua (Beacco, 2010, p. 103).

Desde esta mirada sobre el MCERL, se analizaron los descriptores del nivel A, buscando su aplicación en el diseño de cursos de enseñanza de lenguas indígenas como 
segundas lenguas, es decir, reconociendo que el público a quien va dirigido se compone en su mayoría de jóvenes y adultos alfabetizados, interesados en acceder al conocimiento de una lengua distinta a su primera lengua. Este ejercicio resultó en la acotación de ocho referentes de lengua para un nivel A1, a manera de guía/orientación para el diseño de "programas" de enseñanza en este nivel, que permitan también hacer un balance de los alcances de este Marco para las lenguas nacionales.

Con este propósito se abrió una etapa de planeación y diseño de programas que derivó en su ejercicio como curso piloto de enseñanza. Esto generó que en la dinámica de trabajo, de manera paralela, se planeara la aplicación de los referentes (secuencia didáctica), se diseñaran las actividades y materiales, se aplicaran y evaluaran dichas planeaciones, actividades y materiales. De manera que se conformaron los programas de enseñanza de lengua indígena como segunda lengua del nivel A1, con el conjunto de planeaciones (Material para el maestro) y actividades (Material para el alumno) diseñados, aplicados y reformulados por medio del curso piloto. Las lenguas con las cuales se realizó ese trabajo son: mexkatl (náhuatl de la huasteca), ombeayiüts (huave de San Mateo del Mar), hñahñu (otomí del Valle del Mezquital), bot'una (matlatzinca), sa'an savu (mixteco de Atlatlahuca), werarika (huichol), batsil k'op (tseltal), he hme (chinanteco de San Antonio Analco), con distintos grados de avance en cada una.

Durante el proceso, principalmente en la etapa de diseño y aplicación, cuando los asesores de lengua se enfrentan a elaborar la planeación de la enseñanza -qué competencias se quieren lograr en el estudiante, cómo lo va a lograr, con qué material lingüístico- surgen de manera explícita las implicaciones de "enseñar" una lengua, en particular en relación con el vínculo entre lengua-cultura y su expresión en las prácticas comunicativas en que se concreta el uso de las mismas.

\section{Los "referentes" para la enseñanza de lenguas indígenas}

En el ejercicio que se ha realizado, se retoman los descriptores generales, desglosando su contenido en objetivos comunicativos, objetivos lingüísticos y objetivos culturales. Este conjunto de elementos conforma lo que se denomina "referencial" de enseñanza, es decir, establece los objetivos que se propone que el estudiante sea capaz de lograr en la lengua meta. Para ilustrar lo anterior se presenta el esquema (tabla 1) del primer referente general que se ha construido. 
Tabla 1. Referente 1 de competencias comunicativas de nivel A1 para las lenguas del proyecto.

\begin{tabular}{|c|c|c|}
\hline Nivel: A.1 & \multicolumn{2}{|c|}{ Referente 1: Puede saludar, proporcionar y solicitar a otros sus datos personales. } \\
\hline Descriptores & \multicolumn{2}{|c|}{$\begin{array}{l}\text { - Puede presentarse con frase(s) corta(s). } \\
\text { - Escucha y comprende la situación de saludo y presentación breve. } \\
\text { - Puede relacionar palabras (oral y en pizarrón) con dibujos o ademanes. } \\
\text { - Puede llenar un formulario en la lengua. }\end{array}$} \\
\hline $\begin{array}{l}\text { Situación } \\
\text { comunicativa }\end{array}$ & \multicolumn{2}{|c|}{$\begin{array}{l}\text { Conociéndose, el contexto del salón de clases es ideal para este referente, ya que el } \\
\text { grupo tiene que constituirse y los alumnos conocerse (revisar cómo se hace el contacto } \\
\text { con alguien nuevo en la comunidad de referencia). }\end{array}$} \\
\hline & \multicolumn{2}{|c|}{ Sabrá saludar y presentarse y preguntar a otros sus datos personales. } \\
\hline $\begin{array}{l}\text { Objetivos } \\
\text { culturales }\end{array}$ & \multicolumn{2}{|c|}{$\begin{array}{l}\text { Aprenderá a expresarse de acuerdo con los hábitos comunicativos de habla de la } \\
\text { comunidad. Es importante que conozca las diferentes formas de saludo utilizadas, así } \\
\text { como formas de otras variantes para que ésta no se instituya como una barrera para el } \\
\text { contacto. }\end{array}$} \\
\hline \multirow[t]{3}{*}{$\begin{array}{l}\text { Objetivos } \\
\text { lingüísticos }\end{array}$} & Gramaticales & $\begin{array}{l}\text { pronombres y posesivos de } 1^{\circ} \text { y } 2^{\circ} \text { persona sg. Reverenciales, si existen } \\
\text { en la lengua. }\end{array}$ \\
\hline & Frases & $\begin{array}{l}\text { ¿Cómo te llamas?, Me llamo..., ¿De dónde eres?, Soy de..., ¿Qué } \\
\text { estudias?, Estudio..., ¿En qué trabajas...?, Trabajo en... }\end{array}$ \\
\hline & Léxico & llamar, ser, estudiar (carreras), trabajar (oficios). \\
\hline
\end{tabular}

Fuente: Diseño del proyecto FPEALIL2.

De esta manera se propusieron ocho referentes que, a la luz de las particularidades de la lengua y la cultura que se busca enseñar, pueden ser modificados de una lengua a otra, develando en esas mismas las particularidades del vínculo lengua y cultura inherentes a la enseñanza de una lengua. ${ }^{8}$

Esto puede observarse cuando, para la concreción de tales referentes, el asesor de lengua se enfrenta a la identificación y definición de los “contextos" de uso, esto es a ubicar aquella situación comunicativa, ${ }^{9}$ en la que se podrían realizar las funciones planteadas en los descriptores de competencias. Lo anterior nos remite a la comprensión de las lenguas como prácticas sociales, las cuales dependen de los propósitos (motivaciones y finalidades) de los participantes.

\footnotetext{
${ }^{8}$ Los ocho referentes globales propuestos para el nivel A1 son los siguientes: 1. Puede saludar presentarse y despedirse, 2. Puede describir a las personas de su entorno, 3. Puede hablar de su parentela, 4. Puede hablar de lo que está haciendo, 5. Puede expresar sus hábitos, 6. Puede expresar sus gustos y preferencias, 7. Puede desenvolverse en una compra simple, 8. Puede expresar su estado de ánimo, las razones y preguntar a los otros.

${ }^{9}$ Una situación comunicativa se comprende como el lugar y momento en que ocurren eventos y actos de uso de la lengua, los cuales están regulados social y culturalmente (Hymes, 1974; Pilleux, 2001).
} 
Las prácticas sociales del lenguaje son pautas o modos de interacción que, además de la producción e interpretación de textos (orales o escritos), incluyen una serie de actividades vinculadas con éstas. Cada práctica está orientada por una finalidad comunicativa y tiene una historia ligada a una situación cultural particular. Por ejemplo, las prácticas del lenguaje oral que involucran el diálogo son muy variadas porque éste se establece o se continúa de acuerdo con las regulaciones sociales y comunicativas de las culturas donde tiene lugar. No en todas ellas el diálogo se establece ni se continúa del mismo modo: en algunas culturas indígenas, los niños no deben dirigirse a los adultos o iniciar una conversación sin que primero hayan sido interpelados por ellos; en otras, las mujeres tienen un lenguaje especial para hablar entre ellas (Quinteros y Corona, s. f.).

Sin duda, lo anterior impacta en el diseño de programas y materiales de enseñanza, considerando que la selección de situaciones comunicativas implica también la selección de la imagen o representación existente, la cual se ofrece de la cultura que acompaña a esa lengua, como se aborda más adelante.

\section{Lengua y cultura en los programas de enseñanza de lenguas}

La idea de que la enseñanza de lenguas no es sólo la de sus estructuras o expresiones necesarias para entablar una conversación, empieza a desarrollarse desde hace mucho tiempo con los enfoques comunicativos, los cuales recuperan la noción de que el acto de comunicación se encuentra normado por reglas culturales impuestas por la comunidad de habla, y que una comunicación eficiente que logra su meta, necesita del conocimiento de dichas reglas, planteamientos desarrollados por la etnografía del lenguaje, expuesta por Dell Hymes $(1974,1976)$. Desde entonces se han efectuado debates y elaborado propuestas sobre cómo integrar la cultura en los programas de enseñanza de lenguas.

Si bien, en este texto se hace referencia en especial a los programas de segundas lenguas, para abordar las implicaciones del vínculo entre lengua y cultura en la elaboración de programas de enseñanza, es necesario reconocer la diferencia entre alguien que adquiere una primera lengua en su entorno y alguien que llega a un salón de clases para aprender una lengua adicional o segunda lengua.

\subsection{Primera lengua contra segunda lengua}

Las teorías actuales de adquisición del lenguaje sostienen que la primera o primeras lenguas (ya que éstas pueden ser varias simultáneas) se adquieren de forma inconsciente, es decir, las personas llevan a cabo operaciones de orden lógico que les permiten inducir los principios que regulan las lenguas y sus usos. Es así que los programas que trabajan primera 
lengua, se dirigen a estudiantes que llegan al salón de clases hablando la lengua objeto y manejando las pautas que la regulan.

a través del lenguaje, describe el mundo tal como lo experimenta, pero la misma forma en que lo experimente está informada por el lenguaje que ha aprendido a hablar: la forma en que el lenguaje mismo se estructura gramaticalmente y las cosas que se pueden decir dentro de esa estructura. Incluso sus emociones dependerán de la cultura en la que crece (Claxton, 2001, p. 205).

Gran parte de esa información sobre las pautas sociales para el uso de la lengua no es consciente, tal y como no somos conscientes de las reglas gramaticales que rigen la lengua que hablamos. En general, esto es lo que ofrece la escuela en primera lengua, un desarrollo lingüístico para reflexionar sobre la lengua que se habla y sus usos y, a partir de ahí, aumentar repertorios lingüísticos y usos, como el lenguaje académico.

En segunda lengua, en los niveles básicos, se trabaja con estudiantes que quieren aprender una nueva lengua y sus usos, pues ya poseen una primera lengua con los referentes culturales que conlleva, como menciona Zarate et Gohard-Radenkovic: "los aprendientes, por la lengua que hablan, traen consigo elementos visibles o invisibles de una cultura dada" (citado en Boubakour, 2010, p. 16 ).

La cuestión, entonces, en un programa de lenguas es preguntarse: cómo se trabaja con los sujetos para que se adentren a otro universo cultural haciéndolo accesible, pero sin crear estereotipos.

\subsection{De la definición de cultura en los programas de lenguas}

Ahora bien, para elaborar una propuesta que incluya las particularidades culturales en la enseñanza de una lengua, es necesario explicitar qué se entiende por cultura. Esto ha dado apertura a múltiples debates en las ciencias sociales. En enseñanza de lenguas, sin embargo, el debate se ha dirigido hacia la regulación cultural de la comunicación.

La comunicación es, vista así, como el acto de poner en común las experiencias particulares mediante enunciados, con el fin de establecer acuerdos intersubjetivos sobre el "mundo de todos", el conjunto de mapas que conforman la cartografía que por convención cultural llamamos "realidad". Y la cultura, la paulatina decantación de estos enunciados lingüísticos e icónicos, que en la medida en que son colectivamente asumidos van conformando un humus, un sedimento para uso consciente o inconsciente de todos, tal sedimento es la tradición cultural que empapa a los individuos de modo inevitable, lo sepan o no, lo quieran o no (Chillon, 2001, p. $32)$.

Así, la cultura puede ser entendida, en el campo de la enseñanza de lenguas como el 
mapa que indica u orienta el comportamiento verbal y no verbal que el usuario de la lengua debe tener en una situación o interacción dada. De esta forma, la cultura tutela los usos de la lengua a partir de los cuales se adquieren estructuras lingüísticas (léxico, gramática) para lograr metas comunicativas (pedir, informar, preguntar, etcétera).

Desde esta comprensión de la cultura tiene lugar el enfoque comunicativo -de hecho es uno de sus núcleos fundacionales- y de éste se deriva el diseño de programas de enseñanza, organizado a partir de situaciones y eventos comunicativos, es decir, considerando que el uso de la lengua está intrínsecamente vinculado a las prácticas culturales, sociales y organizativas de sus usuarios, en las cuales toman forma distintos registros y géneros lingüísticos.

Hasta aquí se ha abordado la relación lengua-cultura en la enseñanza de lenguas desde el concepto de comunicación, de la forma en que la lengua se organiza en géneros de intercambio verbal o textual normados culturalmente. No obstante, la lengua representa también el instrumento mediante el cual expresamos el mundo y lo que pensamos de él, es decir, constituye un dispositivo que, de alguna manera, da forma a lo que pensamos a través de los conceptos de que dispone, como lo expresan Chillon (2001) y Claxton (2001). La lengua es también un producto cultural: la forma en que estructura sus enunciados, el léxico de que dispone, son culturalmente distintos de una lengua a otra, es decir, las terminologías del parentesco, los efectos de sentido, los clasificadores numerales, por ejemplo, son elementos que determinan operaciones cognitivas para construir lo "real". Así, los aprendices de una lengua se "acercan", junto con la estructura en uso de la lengua, a esa forma particular de ver el mundo.

Es por estas consideraciones que el abordaje de la "cultura" en la enseñanza de lenguas segundas, no puede reducirse a un dato o a un contenido informativo sobre el país del que se aprende su lengua (Sánchez Lobato, 1999, p. 14). Por el contrario, se requiere tratar la cultura de manera integral en los programas de enseñanza, por consiguiente quien diseña un programa necesita reflexionar sobre esas redes de significado contenidas en las estructuras lingüísticas, y que le pueden parecer a los aprendientes como faltos de sentido, sin que el diseñador lo perciba, puesto que para él fueron significados adquiridos en la participación social (Cole, 1999) (de forma inconsciente) fuera del aula, ya que la cultura también nos es transmitida sin que nos percatemos de cuáles son sus componentes.

Por ello, quien se da a la tarea de diseñar los contenidos de enseñanza de una lengua particular, lleva a cabo un trabajo de reflexión sobre las características estructurales, comunicativas y culturales de su lengua, no para "explicarlos de forma declarativa", sino para 
diseminarlos y permitir su interpretación, darles sentido. Esto se refleja en que los programas, los "diseños" de los programas de distintas lenguas, se van diferenciando y en momentos, incluso, alejando.

\section{Lengua y cultura en la elaboración de materiales para la enseñanza de las lenguas originarias}

La tarea de incorporar contenidos culturales en los materiales para la enseñanza de una lengua, desde un enfoque comunicativo, busca vincular la lengua-cultura de manera integral y gradual. Si bien, es posible integrar información explícita sobre la cultura del pueblo que habla la lengua que se pretende enseñar, esto requeriría, en los primeros niveles, presentar la información en la lengua del estudiante -en el caso de México, en español-, haciendo inviable que las clases de lengua ocurran, prioritariamente, en la lengua meta.

En esta perspectiva, es posible sugerir que, desde un enfoque comunicativo, el abordaje de la cultura en la elaboración de materiales para la enseñanza de la lengua, se puede integrar a través de tres instancias:

a) El diseño y la organización del programa, considerando las prácticas comunicativas, las situaciones y los eventos propios a la comunidad que habla esa lengua.

b) La cosmovisión, expresada en las redes de significado contenidos en las estructuras lingüísticas y que toma forma en el léxico, los efectos de sentido, las pautas discursivas, etcétera.

c) La información explícita que se proporciona en los textos, a manera de cápsulas informativas que se integran en los materiales.

Todas estas instancias se encuentran interrelacionadas en, como diría (1996), telarañas de significado, y es necesario un diseño que permita negociar significados entre la lengua que se enseña y los referentes del aprendiente, lo que Breen (1987) denomina tender puentes.

Para ejemplificar los retos que implica integrar la cultura en el diseño de un programa, se retoman los dos primeros referentes de competencias de los cursos: Puede presentarse y presentar a otros y Puede hablar de su parentela, que conformaron una unidad de aprendizaje. 
En las diversas culturas difieren las formas de entablar contacto con las personas, las convenciones de saludarse o presentarse, así como las pautas de cortesía, están normadas por su cultura. En la tradición de presentarse en algunas culturas, se empieza diciendo el nombre, procedencia y algunos otros datos personales, los cuales no aplican en muchas otras, sobre todo en aquellas lenguas que sólo se usan en proximidad, como las lenguas indígenas, por lo que todos se conocen y aquellos que no, se preguntarán por la parentela, más que por la persona en sí misma. Pero en un salón de clases o un espacio de enseñanza de una lengua, cuando los estudiantes provienen de diferentes lugares, tienen que aprender a presentarse de alguna manera, además, el salón de clases es un espacio con sus propias necesidades de comunicación, un contexto en sí mismo, ¿cómo conjuntar dos tradiciones comunicativas diversas? En los diseños de los programas, la clase de "presentarse" se ha convertido en la clase de "conocerse", la cual busca no calcar una forma que no corresponde a la lengua meta, al introducir de inmediato la parentela para que los estudiantes entiendan que fuera del salón de clases, en la comunicación con los hablantes en la comunidad, tendrán que usar estas formas y no las practicadas en el aula. Aunque esto parece muy simple, tiene implicaciones complejas en el diseño. Revisemos dos casos.

En el pueblo tseltal, al preguntar por alguna persona, se pregunta por un grupo doméstico y más precisamente por el jefe de éste. Sin embargo, la "familia" adquiere en cada cultura una construcción particular, ninguna lengua de las que se trabajaron posee un término que recubra con exactitud lo que en español denominamos "familia". En esta lengua, lo más cercano al término "familia" es k'opojan, que refiere a hogar. Este pequeño núcleo, sin embargo, se encuentra inserto en agrupaciones mayores, los linajes jol biil y los clanes tijinabal. Así, cuando se conocen personas de diferentes comunidades, se preguntan por su clan, incluido en el apellido o/y por su linaje. El diseño para incluir k'opojan, jol biil y tijinabal en la unidad de aprendizaje sin tener que explicitarlo, se llevó a cabo en varios momentos; primero se abordó el núcleo reducido más cercano al aprendiente, y una vez afianzado se extendió a las dos unidades mayores, para lo cual se realizó la imagen 1. En sus cuadros podemos observar los núcleos reducidos k’opojan, los linajes "jol biil”, que puede ser "chab" 'abeja' o "jol chij" 'venado', entre otros, y estos dos contenidos en tijinabal. Gómez o tijinabal Santiz 
Imagen 1. Jool biil y tijinabal.

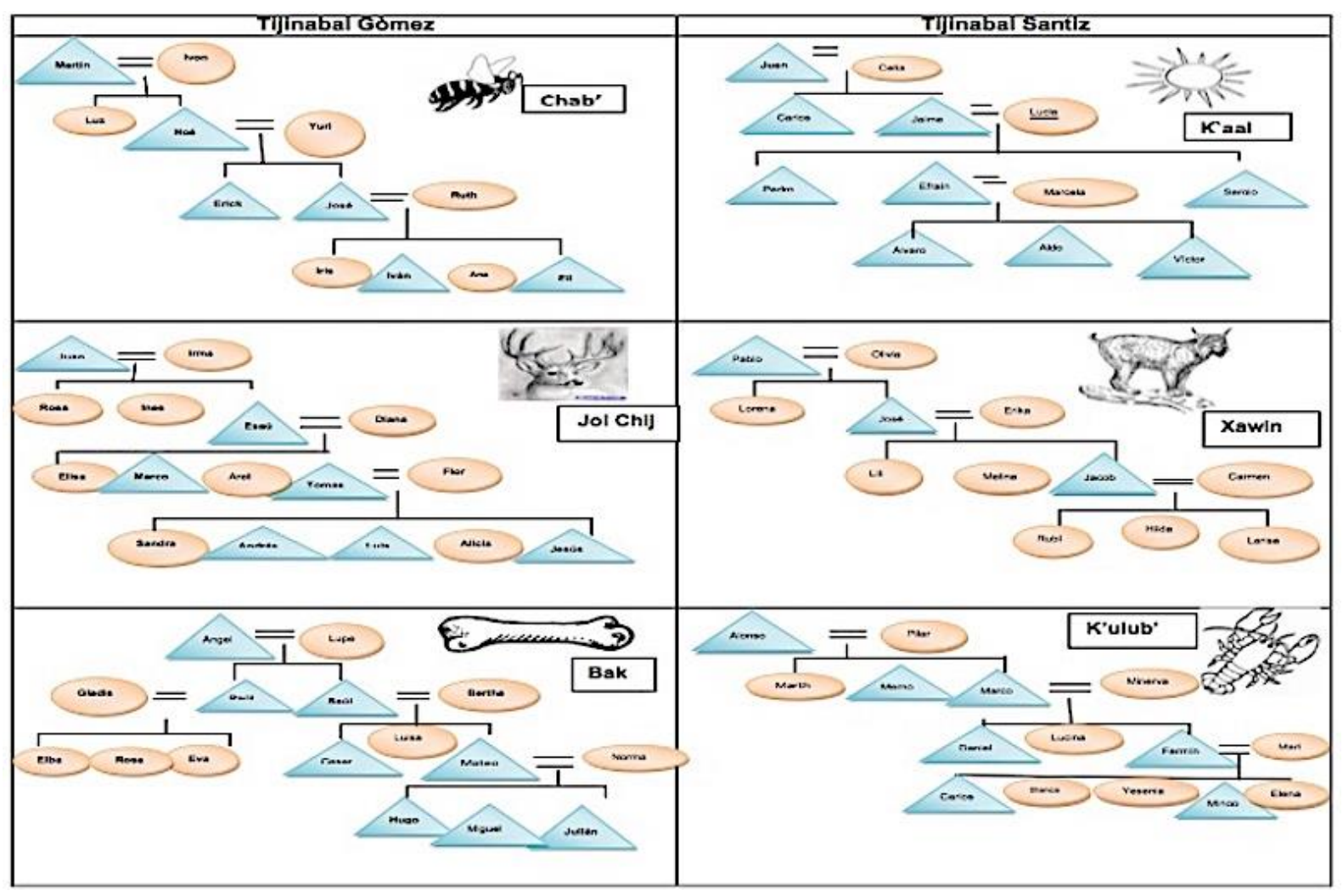

Fuente: Diseño de Inés Gómez Sántiz.

De esta forma, los estudiantes entienden a qué se referirán las personas cuando les pregunten por su jool biil o su tijinabal. Esto se encuentra muy alejado de la concepción de un hispanohablante y presenta cierto grado de dificultad, sobre todo porque no es algo que el que aprende la lengua batsil k'op (tseltal) pueda usar para hablar de su familia, puesto que no hay clanes ni linajes; sin embargo, quien aprende tseltal necesita conocer cómo se expresan esas relaciones en esta lengua, y poder decir, -no tengo linaje- "maku jool biil". Negociar significados. Pero esta diferencia en la estructura del parentesco tiene otras implicaciones, por ejemplo léxicas. La parentela tseltal es patriarcal y patrilineal, lo que significa que cuando la mujer se casa pasa a pertenecer al clan del marido, por lo que para ellos es importante reconocer el lado materno del paterno, lo que hacen con la nomenclatura, el tío materno tiene una denominación diferente a la del tío paterno. Así que en una conversación el aprendiz tiene que utilizar el término adecuado para no producir confusiones, esto significa darle sentido a esta diferenciación y, en esa medida, entender la cultura.

Imagen 2. Línea paterna y línea materna en la parentela tseltal. 


\section{Swaxukebal at'elil 8. jilawilme!}

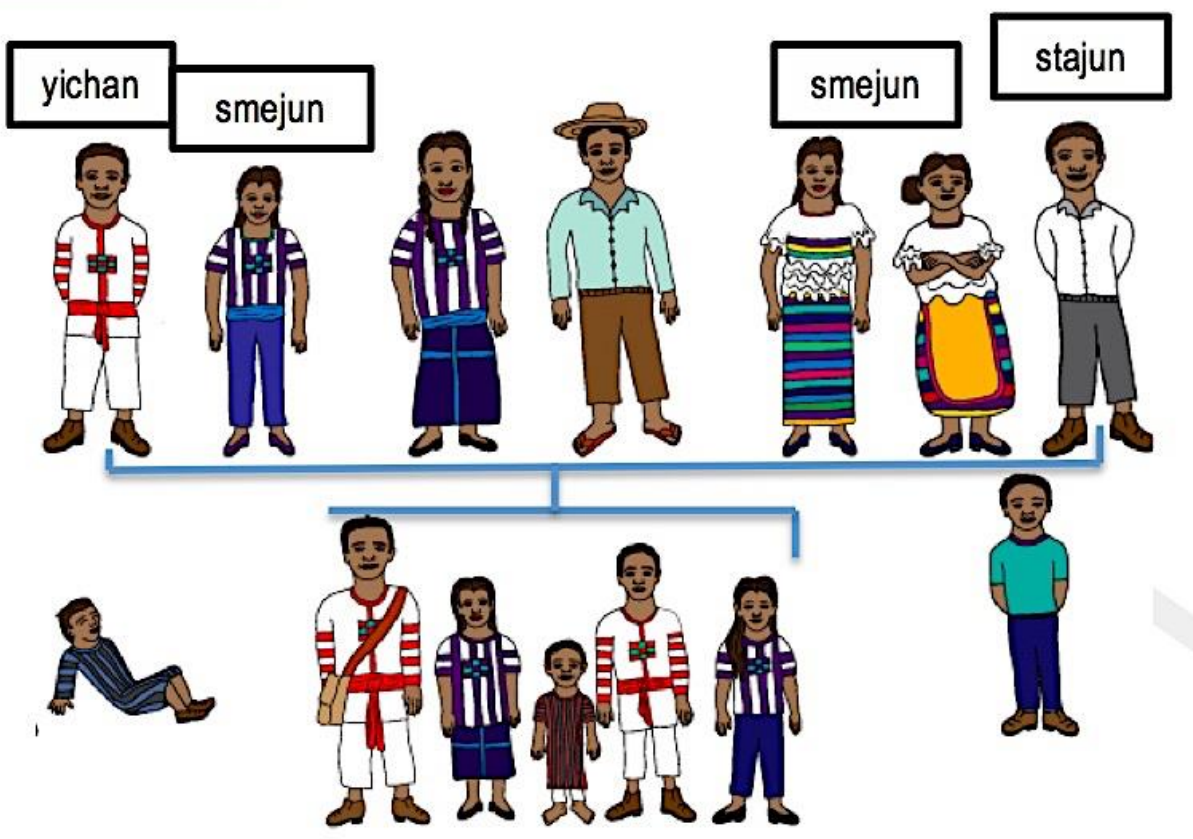

Fuente: Diseño de Rodrigo Padilla López.

Para esta misma meta comunicativa, la lengua ombeayiüts (huave) generó un diseño diferente. Aunque ambas lenguas comparten los usos de la situación comunicativa para conocerse, la estructura del parentesco es diferente; la palabra que puede referir al núcleo restringido “familia' o más bien hogar es monkiül saniüng, 'los que habitan una casa', pero ésta sólo considera a la descendencia directa y fuera de ella se usa el término sakwalaats, que incluye a la familia política y su descendencia. Para precisar a estos últimos se utiliza una terminología muy compleja, por ejemplo para tío, minoj michiig nüx ximüm, que en su traducción literal significa 'esposo de hermana menor mujer, de mi madre', o bien para primo, mikwal nej michiig nüx ximüm, 'hijo él hermana menor mujer de mi madre'. 
Imagen 3. Akwalaats.

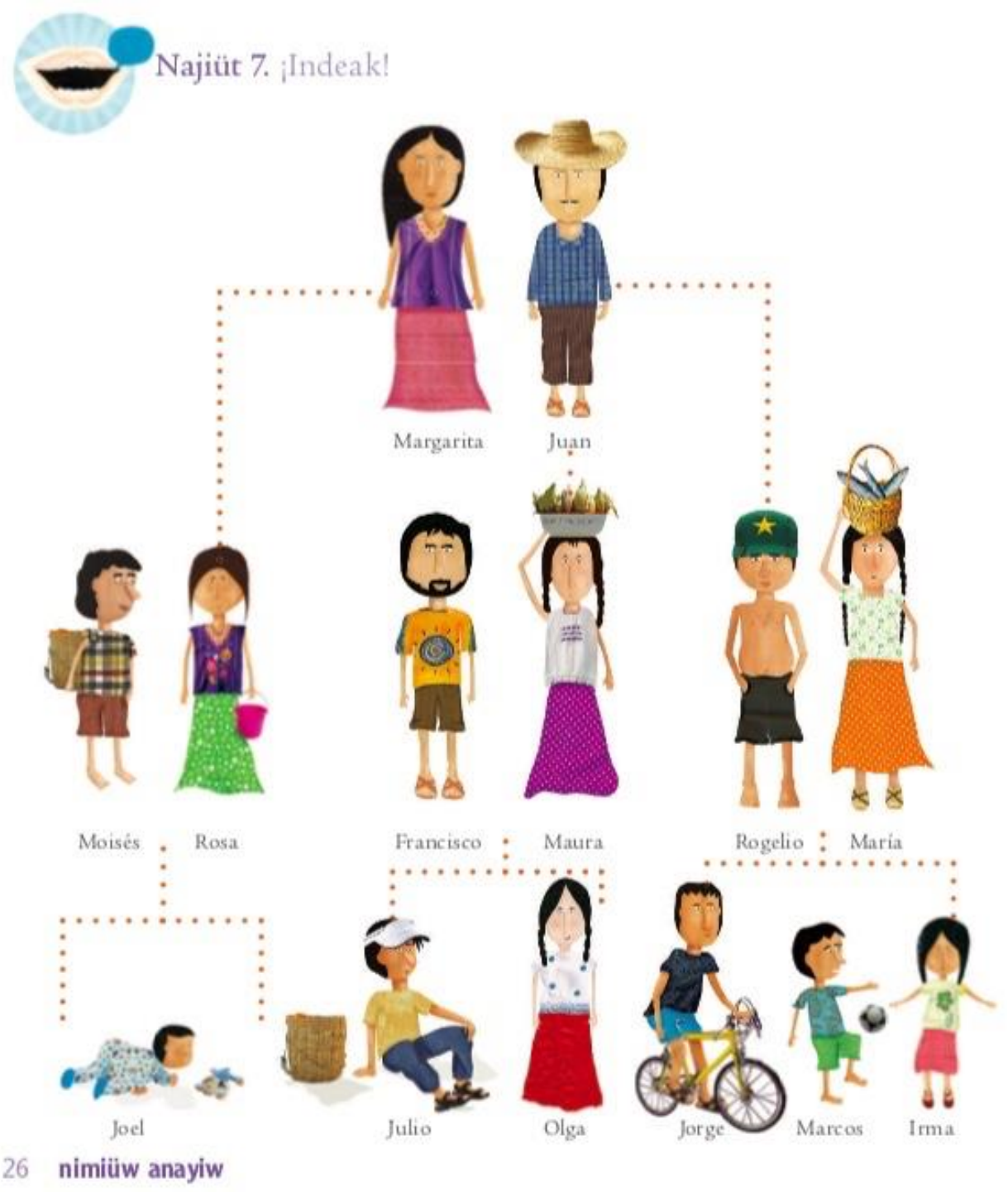

Fuente: Ilustración de Salvador Jaramillo.

A partir de estos dos ejemplos se puede observar cómo la cultura y sus particularidades se hacen patentes en la elaboración de un programa de enseñanza de la lengua, donde se encuentran integrados, no se pueden separar. Igualmente hablan de que no se transfiere un modelo, sino que cada lengua requerirá diseños diferentes e incluso tiempos diferentes para aplicarlos. Esta meta comunicativa en ombeayiüts (huave) requirió tres sesiones, mientras que en batsil k'op (tseltal) se trabajó en seis.

Como se ha visto en el apartado anterior, la estructura de la lengua lleva en sí misma información de una cultura, sin embargo, en el diseño de programas, la estructura de la lengua es un elemento que no se selecciona, es obligada, lo que se selecciona son los contextos 
comunicativos que se abordan, no se puede presentar toda la gama de eventos, sería una tarea imposible. Conforme el material se va presentando, los estudiantes comparan los referentes de significado con su primera lengua u otras que conozcan y cómo funciona de manera similar o diferente. Por ejemplo, si van a contar en lenguas ombeayiüts (huave) o batsil k'op (tseltal), tienen que conocer los clasificadores numerales, sin lo cual no podrían contar de forma adecuada en la lengua meta.

Imagénes 4 y 5. Clasificadores numerales de la lengua ombeayiüts (huave)

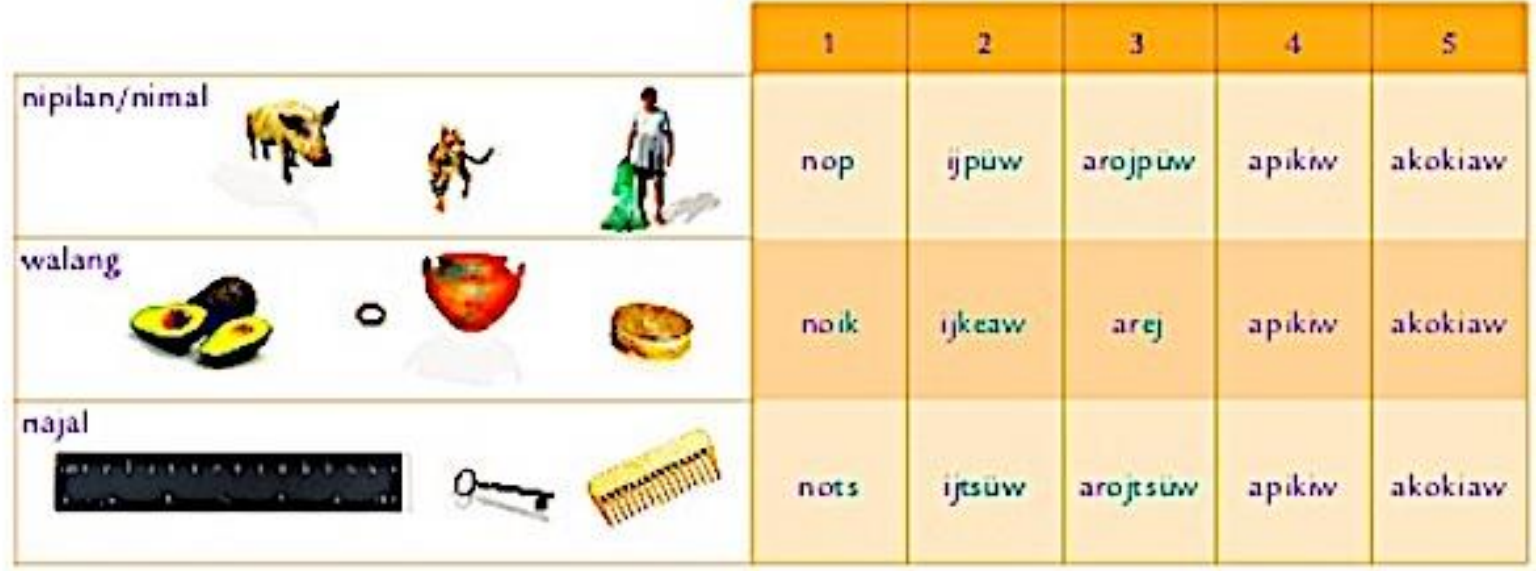

Fuente:, diseño de Salvador Jaramillo

Imagen 5. Clasificadores numerales de la lengua batsil k’op (tseltal).

Swakebal at'elil 6. jilawilme!

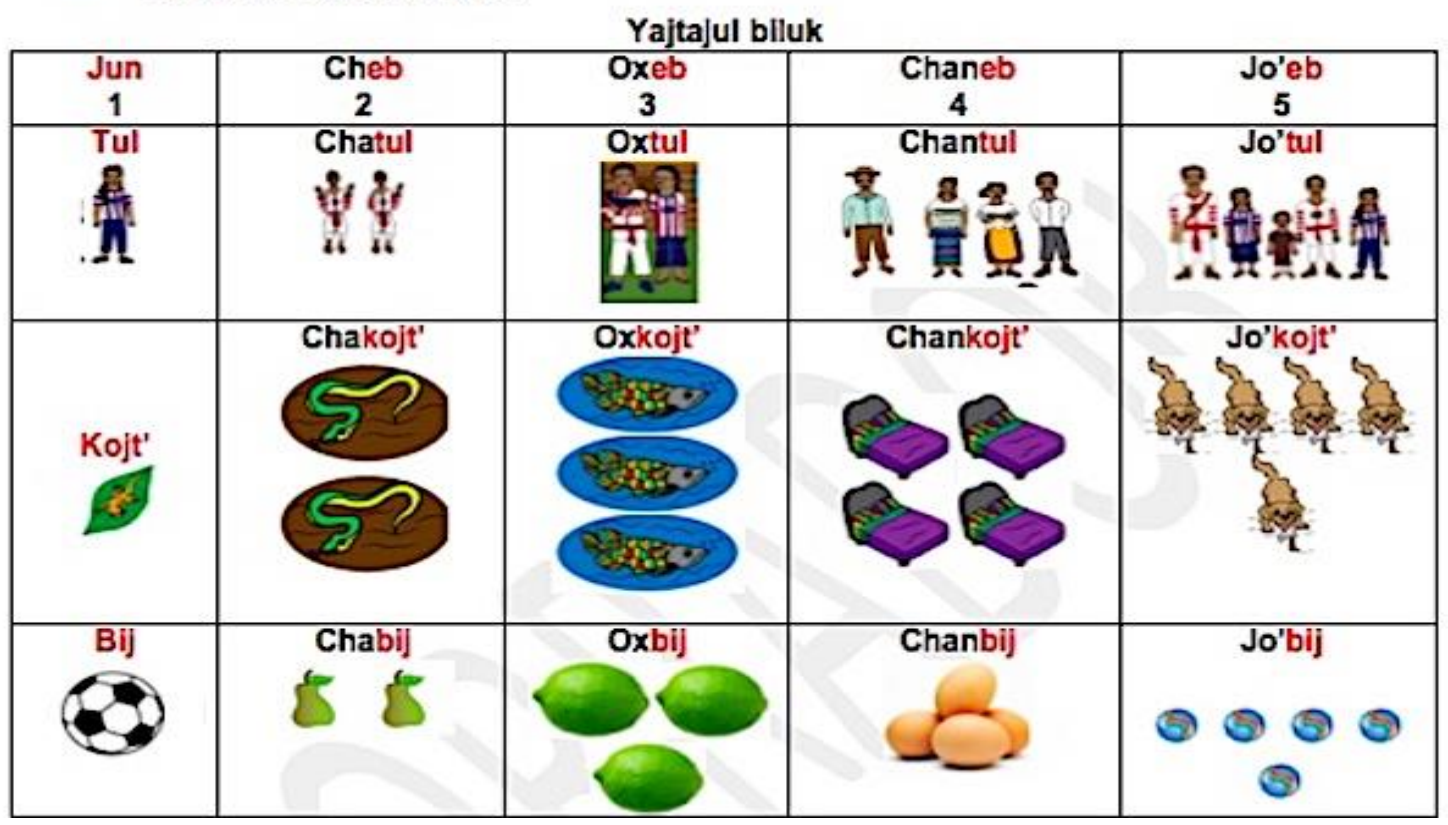

Fuente: diseño de Rodrigo Padilla López.

En las imágenes 4 y 5 se observa que para expresar números en estas lenguas se debe 
especificar el tipo de elemento que se cuenta, ya sean con recursos léxicos o afijos. Esta información podría describirse explícitamente en la lengua de los estudiantes, sin embargo desde el enfoque comunicativo se busca que se asocie en el uso, para expresar algo. Una explicación nos llevaría, tal vez, a hacer una interpretación inexacta, mientras que aprendiendo a usarla, los alumnos internalizan esta información, la comparan con su lengua, la interpretan y sacan sus conclusiones-

Esta forma de acercarse a la lengua aporta al estudiante información que puede aplicar en otros contextos. Por ejemplo, si tiene que contar en batsil k'op (tseltal) otro tipo de objetos que no están en el ejercicio, cuenta ya con una idea del uso de los números y sus clasificadores. Es tal vez la parte de la cultura que, extendiendo la enseñanza de las lenguas originarias hacia nuevo público, puede aportarnos otras miradas del mundo.

Los contenidos culturales explícitos son tal vez los más difundidos. Incluso es posible tener clases sobre cultura e historia de un pueblo determinado. No obstante, desde el enfoque y los objetivos que adoptamos para la enseñanza de la lengua, los contenidos culturales también se trabajan en la lengua meta, buscando graduar la dificultad que puede representar para el estudiante.

En general, la revisión de las prácticas culturales de los pueblos se enseña en cursos para especialistas, antropólogos y otros interesados. No obstante, en la enseñanza de lenguas es necesario partir de las prácticas culturales en que se materializa el uso de la lengua, de otra manera ¿cómo se puede entender una lengua sin estas prácticas? Por ejemplo: la manera en que se segmenta el tiempo, o como se abordará a continuación, la forma de entender la salud, la enfermedad y las prácticas curativas asociadas a ello.

Una de las metas comunicativas que se definieron establece que el estudiante pueda expresar su estado de ánimo y su estado de salud. Pero, ¿cómo se expresa esto en lenguas, como el ombeayiüts (huave) o el batsil k’op (tseltal), cuando el estado emocional está asociado a la salud?

En ombeayiüts (huave) la expresión 'estoy alegre' se dice najneaj ximeaats, y 'me duele el estómago’ se dice nekoy ximeaats; en ambos casos se utiliza la palabra ximeaats, que corresponde a, algo así, como 'mi interior'; nekoy está formado por un marcador de verbos estativos ne- y el nombre koy, que en español refiere a 'conejo' o 'calambre', algo así como 'aconejarse' o 'acalambrarse'. Esta información sobre la terminología no se explica al estudiante en español, se sobreentiende con la estructura de la lengua que el estudiante va deduciendo. Lo que se explicita son las prácticas usadas para curarse del 'aconejamiento' o el 
'acalambramiento', para lo cual se diseñó el material que se presenta en la imagen 6.

Imágen 6. Comprensión lectora sobre prácticas de curación y prácticas curativas en la lengua ombeayiüts.

Najiüt 4. ;Iteow!
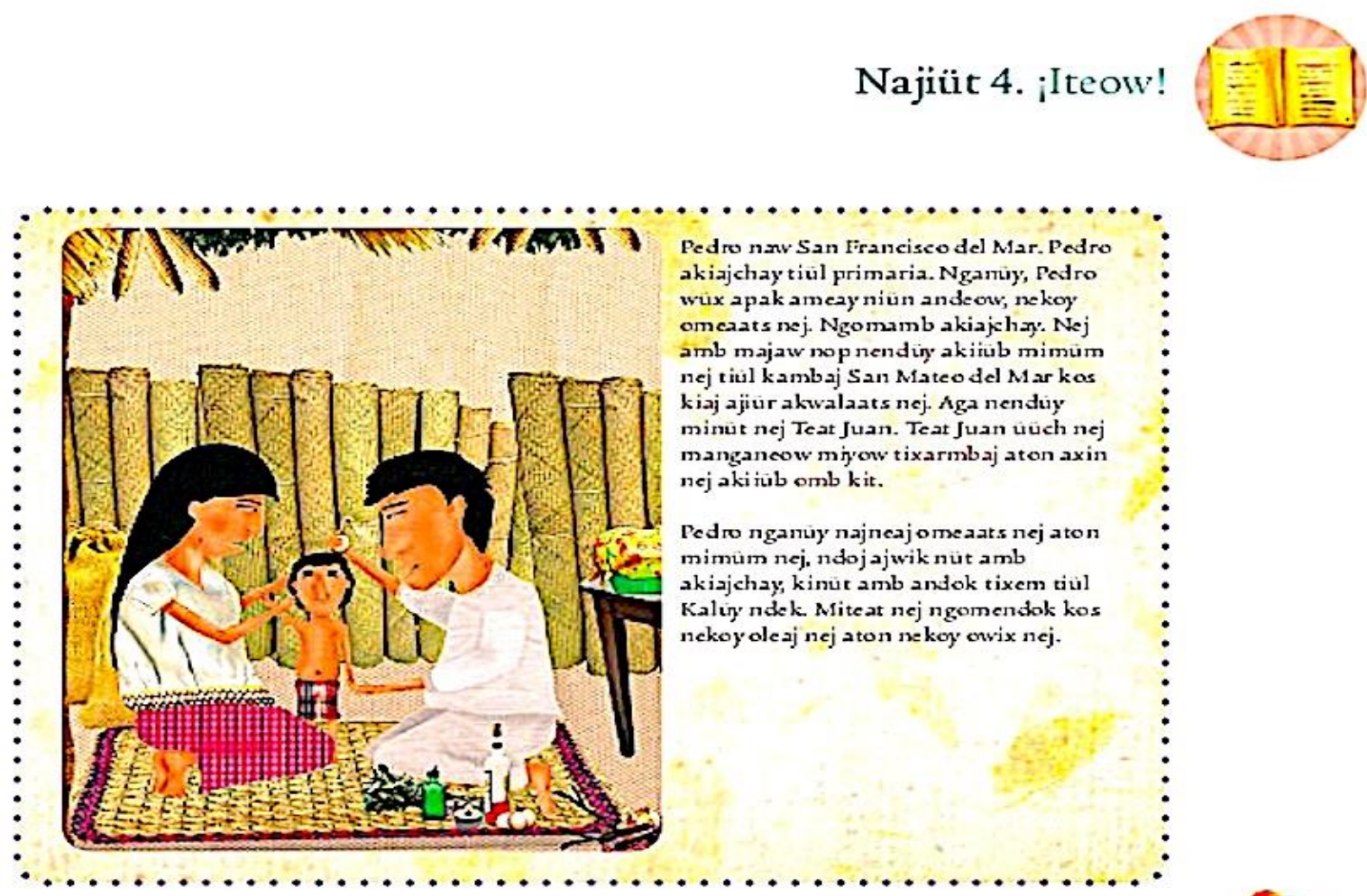

¡I rang! Leaw iteow, nganüy irang nimiüw gajpowüw tengwüy ikmiüw poch

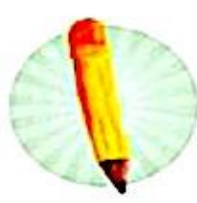

Najiüt 5. ¡Ijaw!

Nipilan

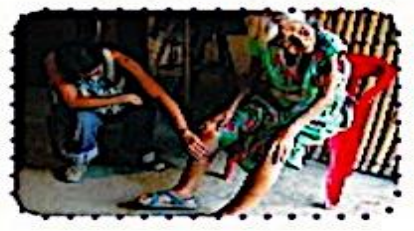

asooig olaats

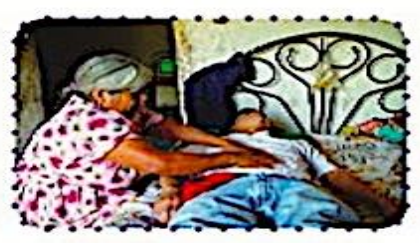

axinotüngeraı

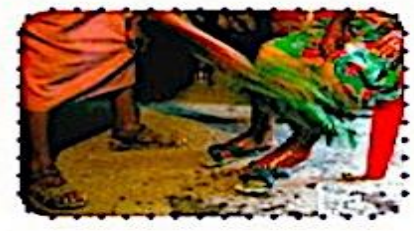

axin aki iüb op najmbeal

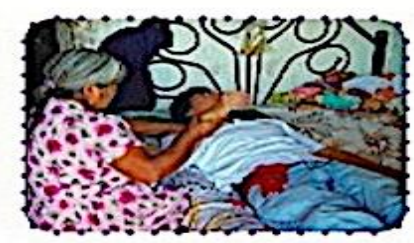

andüy xiüt

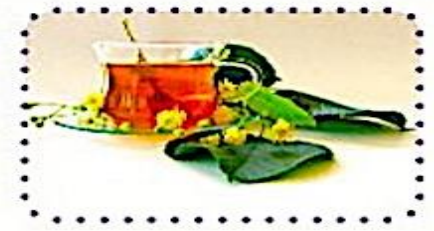

miyow morpeat

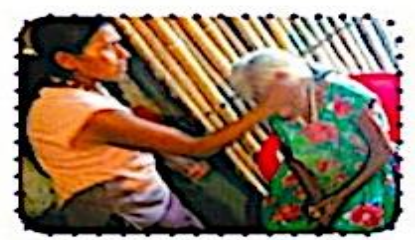

axin akiiüb omb kit

Fuente: Diseño de Salvador Jaramillo. 
En la imagen 6 se observa un texto de comprensión lectora, el cual relata cómo un niño que estaba triste Ngomajneaj omeaats nej, porque le dolía el estómago nekoy amyats nej, es llevado por su mamá a un curandero y cómo éste lo cura con aguardiente, huevo y un té. En la imagen 7 se proporcionan elementos de otras formas de curación que el maestro de lengua puede referir según lo que tenga el enfermo, para que el estudiante pueda entender de qué se está hablando y, de forma autónoma, utilice esa información en la comprensión y producción de textos orales y escritos. La información explícita se va dando para que los alumnos conozcan las prácticas culturales y dónde podrán usar la lengua. En esta parte se necesita también abordar la relatividad cultural, ya que muchas de estas prácticas han sido "satanizadas" o vistas como supersticiones; sin embargo, la intención es que los estudiantes se queden con la idea de que desde la lengua y la cultura ombeayiüts (huave), la curación no sólo se refiere a la atención del cuerpo exterior, sino también a su "interior".

El diseño de los materiales de enseñanza, también pueden incorporar "nuevos" contextos para el uso de la lengua, abonando a la idea de que la lengua "sirve" para expresarse más allá del ámbito comunitario, en cualquier otro espacio, como en el ejercicio de la imagen 8, en el que los aprendices escriben en Facebook, en lengua he hme (chinanteco).

Imagen 8. Ejercicio de expresión escrita en lengua $h \notin h m \notin$.

\section{Ta 5. ¡Hm we' kyun 'ya kyani ¿En thun 'nu?}

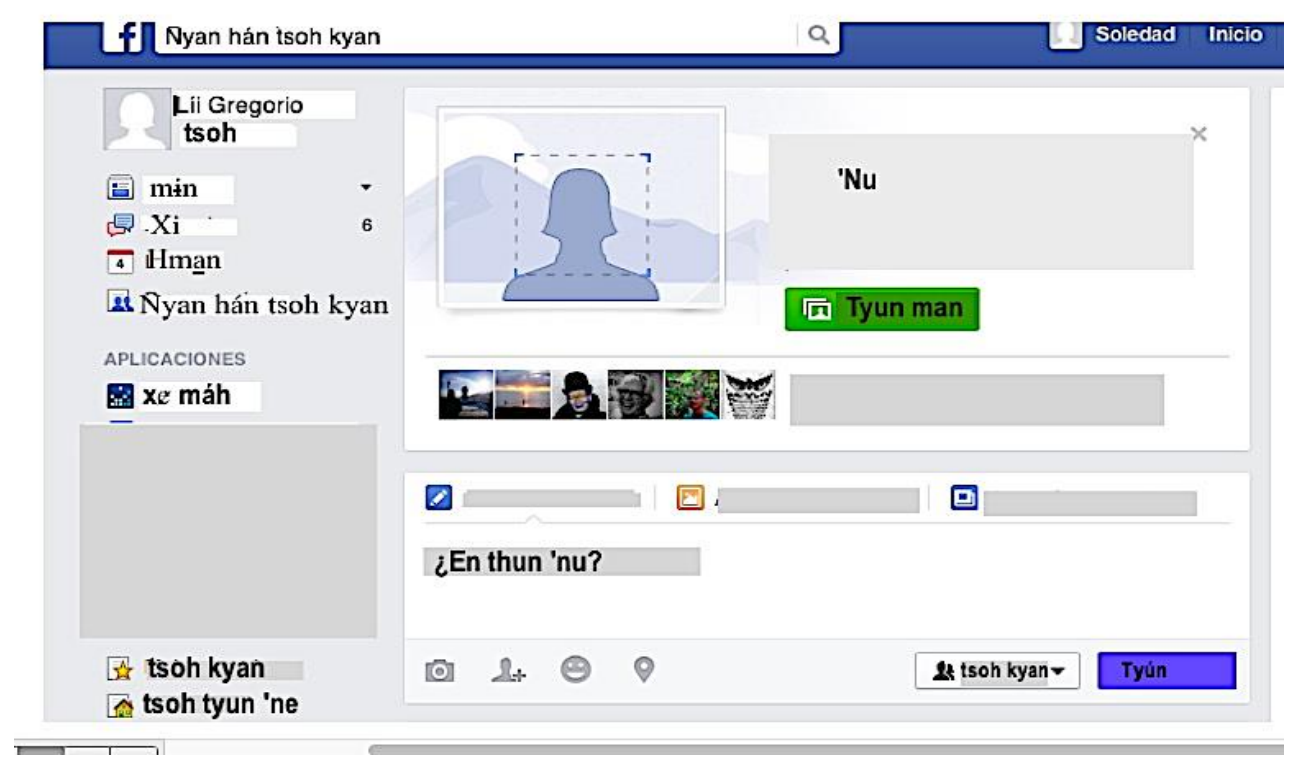

Fuente: Diseño de Alicia Gregorio. 
En este sentido, es posible buscar que las situaciones comunicativas a través de las cuales se hace viable el logro de metas comunicativas, integren nuevos contextos, al mismo tiempo que se van abordando los contextos de uso tradicionales.

Para finalizar, cabe considerar también algunas de las interrogantes que han surgido. Como se ha mencionado, se definieron ocho referentes para iniciar el diseño del primer nivel de lengua, pero no todos se han podido aplicar en la totalidad de las lenguas. Por ejemplo, el caso del referente 6, Puede expresar sus gustos y preferencias. Las lenguas ombeayiüts (huave) y hñahñu (otomí variante EM) no poseen un verbo "gustar", en este contexto se usa el verbo “querer”. Entonces, ¿se transforma la función comunicativa?, ¿expresar lo que me gusta y lo que quiero es lo mismo?, ¿se logra la misma meta comunicativa?, ¿en las culturas citadas "gustar" y "querer" se recubren? Pero no sólo hay que detenerse en lo que "no hay", sino cuestionarse sobre usos y funciones comunicativas que no existen en las lenguas, sobre las que se han elaborado programas de larga tradición de enseñanza, lo que representa todo un programa de investigaciones para trabajar niveles más avanzados.

\section{Conclusiones}

De lo expuesto hasta ahora, si bien no es posible plantear conclusiones finales acerca de la utilidad del MCERL para la elaboración de programas de enseñanza de las lenguas indígenas en México, existe un balance positivo de sus orientaciones como herramienta, cuyos resultados son palpables en el trabajo que desarrollan los enseñantes y las habilidades comunicativas en los aprendices de los cursos piloto. Se ha dado un importante cambio en la visión de "enseñar/aprender" una lengua, y se ha definido como un objetivo viable hablar en la lengua meta.

Quedan muchas preguntas y debates por abordar, en particular desde la vigilancia y el cuidado de respetar la vinculación entre lengua-cultura en el diseño y elaboración de los programas y materiales de enseñanza. Es esta relación, se propone garantizar que no se traslapen funciones y contextos comunicativos desde lenguas mayoritarias a partir de la utilización de los modelos generales, como el MCERL, pero es también necesario responder al reto de la ampliación de contextos de uso de lenguas históricamente minorizadas.

$\mathrm{Y}$, por supuesto, es necesario distinguir públicos y contextos, ya que los programas que hemos referido están dirigidos a público general, que se ubica por lo general en contextos urbanos, lejos de las comunidades de habla y que desea aprender lenguas indígenas nacionales 
en cursos presenciales.

\section{Referencias}

BEACCO, Jean Pierre. Tâches, compétences de communication et compétences formelles. En Politiques publiques et changements en éducation: pour un enseignement réciproque du portugais et du français, Synergies Brésil, Sylvains les Moulins (France), n. 1, p. 97-105, 2010. Recuperado de https://gerflint.fr/Base/BresilSPECIAL1/titre_bresil.pdf.

BOUBAKOUR, Samira. L'enseigment supérieur des langues-cultures: dimensions et perspectives. Synergies Algérie, n. 9, p. 13-26, 2010. Recuperado de https://gerflint.fr/Base/Algerie9/boubakour.pdf.

BREEN, Michael. (1987). Paradigmas actuales en el diseño de programas de lenguas. Comunicación, lenguaje y educación, trad. Fundación Infancia y Aprendizaje, v. 7, n. 8, p. 732, 1987. DOI: https://doi.org/10.1080/02147033.1990.10820940. Recuperado de https://cvc.cervantes.es/ensenanza/biblioteca_ele/antologia_didactica/enfoque01/breen01.htm.

CLAXTON, Guy. Aprender. Barcelona: Paidós, 2001.

CONSEJO DE EUROPA. Marco común europeo de referencia para la enseñanza de lenguas. Enseñanza aprendizaje y evaluación. Madrid: Ministerio de educación, cultura y Deportes, 2001. Recuperado de https://cvc.cervantes.es/ensenanza/biblioteca_ele/marco/cvc_mer.pdf.

CHILLON, Alberto. El "giro lingüístico" y su incidencia en el estudio de la comunicación periodística. Cuadernos de información, n. 14, p. 24-47, 2001. Recuperado de http://www.accionaudiovisual.uc.cl/prontus_fcom/site/artic/20050410/pags/20050410221626. html.

EDUSCOL. Cadre européen commun de référence pour les langues. Ministère de l'éducation nationale et de la jeunesse, 2011. Recuperado de http://eduscol.education.fr/cid45678/cadreeuropeen-commun-de-reference.html.

GEERTZ, Clifford. La interpretación de las culturas, trad. Alberto L. Bixio. Barcelona: Gedisa, 1996.

HYMES, Dell. Hacia etnografías de la comunicación. En Yolanda Lastra y Paul Garvin (comps.). Antología de estudios de etnolingüistica y sociolingüística. México: UNAM, 1974.

HYMES, Dell. La sociolinguiística y la etnografía del habla (115-149). En E. Ardener (ed.). Antropología social y lenguaje. Buenos Aires: Paidós, 1976.

INSTITUTO NACIONAL DE GEOGRAFÍA Y ESTADÍSTICA (INEGI). Censo de Población y Vivienda 2010. Tabulados del cuestionario básico. Recuperado de https://www.inegi.org.mx/contenidos/programas/ccpv/2010/tabulados/Basico/05_02B_MUNI CIPAL_15.pdf.

Quinteros Sicurano, Gabriela y Corona Caraveo, Yolanda. Las prácticas sociales del lenguaje 
en contextos indígenas. Proyecto de investigación. Departamento de educación y Comunicación. México: UAM, s. f. Recuperado de http://www.uam.mx/cdi/pdf/p_investigacion/practicas_sociales.pdf.

PILLEUX, Mauricio. Competencia comunicativa y análisis del discurso. Estudios Filológicos, n. 36, p. 143-152, 2001. DOI: http://dx.doi.org/10.4067/S007117132001003600010. Recuperado de https://scielo.conicyt.cl/scielo.php?script=sci_arttext\&pid=S0071-17132001003600010.

SÁNCHEZ LOBATO, Jesús. Lengua y cultura. La tradición cultural hispánica. Carabela, n. 45, p. 5-26, 1999. Recuperado de https://dialnet.unirioja.es/servlet/articulo?codigo=7206681.

Submetido em 28 de junho de 2021. Aceito em 05 de abril de 2021.

Publicado em 16 de julho de 2021. 